\title{
An Effective Pre-processing Technique for Robust ESPRIT-Based Single-Tone Frequency Estimation against an I/Q Mismatch
}

\author{
Sangdong Kim ${ }^{1}$, Bong-seok Kim ${ }^{1}$, Daegun $\mathrm{Oh}^{1}$, Jonghun Lee ${ }^{1}$ \\ ${ }^{I}$ Advanced Radar Technology (ART) lab., Robotics System Research Division, \\ Daegu Gyeongbuk Institute of Science \& Technology, \\ 50-1 Sang-Ri, Hyeonpung-Myeon, Dalseong-Gun, Daegu, 711-873, South Korea \\ jhlee@dgist.ac.kr
}

\begin{abstract}
This letter proposes an effective pre-processing technique for robust estimation of signal parameters via rotational invariance techniques (ESPRIT)-based single-tone frequency estimation against an $I / Q$ mismatch. For the implementation of a high-accuracy ranging/radar system, parametric-based algorithms such as MUSIC, ESPRIT and their variations have been adopted due to their superior estimation capability for single-tone frequency estimations. Since most ranging/radar system have the direct conversion architecture, $I / Q$ imbalance problem occurs in the form of spurious frequencies of the frequency spectrum. For this reason, the proposed pre-processing technique is designed to make use of the Hilbert transform structure for received signals. The estimation performance of the proposed preprocessing technique in single-tone frequency is derived and compared with the results from a Monte-Carlo simulation. The root-mean-square error (RMSE) of the proposed method is compared with that of an ESPRIT-based method for various parameters.
\end{abstract}

Index Terms-ESPRIT, pre-processing technique, singletone frequency, Hilbert transform, I/Q mismatch.

\section{INTRODUCTION}

Single-tone frequency estimation is one of the main challenging in wireless systems [1], [2]. It has numerous applications in the instrumentation and measurement issues such as testing of sine waves and the single-target detection of the radar. Specifically, millimeter-wave wireless systems (30 $\mathrm{GHz}$ to $300 \mathrm{GHz}$ ) have recently become increasingly widespread for radar systems [3] such as frequency modulated continuous wave (FMCW) radar. In the millimeter-wave systems of the FMCW radar, direct conversion types of architecture are commonly implemented to reduce the cost and power consumption of the receiver. However, because the conventional direct conversion architecture is sensitive to local oscillation and the matching between the components of the in-phase (I) and quadrature (Q) channels, they have especially an effect to DC offset and I/Q imbalance [4]. Although there have been many efforts to improve the accuracy of the single-tone parameter estimation

Manuscript received April 14, 2015; accepted September 15, 2015

This work was supported by the DGIST R\&D Program of the Ministry of Education \& Science Technology, Korea (15-RS-01). process in the presence of AWGN, this problem has not been addressed in conjunction with the I/Q mismatch problem. The I/Q imbalance problem strongly affects the signal processing part when estimating parameters.

For frequency estimations, there are parametric and the non-parametric approaches. Well-known parametric methods are multiple signal classification (MUSIC) [5], matrix pencil [6], Propagator [7] and estimation of signal parameters via rotational invariance techniques (ESPRIT) [8]. The non-parametric methods include the discrete Fourier transform (DFT), the Blackman-Turkey method and the Bartlett method. The performances of the parametric methods are superior to those of the non-parametric methods. Among the parametric methods, ESPRIT achieves quite good performance and is thus widely used. In this letter, we propose an effective preprocessing technique for robust ESPRIT-based single-tone frequency estimation against instances of I/Q mismatch.

\section{Signal Model}

The chirp signal is used widely for millimeter-wave systems such as radar [3] and communication systems [9] because a received chirp signal can be changed to a sinusoidal signal by a de-chirping process.

\section{A. Received Signal without I/Q Imbalance}

In FMCW radar, the transmitted baseband chirp used for time delay estimation is obtained from the models as

$$
s(t)=\left\{\begin{array}{cc}
\cos \left(\left(\omega_{c}+\omega_{s}\right) t+\frac{\mu}{2} t^{2}\right) & \text { for } 0 \leq t<T_{\mathrm{sym}}, \\
0 & \text { elsewhere }
\end{array}\right.
$$

where $\omega_{\mathrm{c}}$ denotes the carrier frequency, $\omega_{\mathrm{s}}$ is the initial frequency, is the rate of change of the instantaneous frequency of a chirp symbol and $T_{\text {sym }}$ is the signal period of a chirp symbol. Assuming a time-invariant channel over $T_{\text {sym }}$, the received signals, perturbed by additive white Gaussian noise (AWGN), can be represented by

$$
y(t)=a s(t-\tau)+z(t)
$$


where $z(t)$ is the AWGN with noise power $\sigma^{2}$ and $a$ and $\tau$ are the complex amplitude and time delay of the received signal, respectively.

To apply parametric-based algorithms such as ESPRIT and MUSIC, de-chirping is done to transform the received chirp signals into sinusoidal signals. In practice, de-chirping is defined as the conjugate multiplication of the received chirp signals and the reference chirp signals $r(t)$ as in ([9], (9)), such that

$$
d(t)=y(t) r^{*}(t)
$$

where ()$^{*}$ means the phase conjugation, $d(t)$ indicates that $y(t)$ is transformed into exponential form. Eq. (3) shows that the transformed signals $d(t)$ as in ([9], eq. (10)) after lowpass filtering have a sinusoidal waveform such that

$$
d(t)=a e^{j \eta(t)}
$$

where $\eta(t)=-\tau t-\omega_{\mathrm{s}} \tau+/ 2 \tau^{2}$. At this point, the time delay of arrival (TOA) parameter $\tau$ is changed to the frequency components of the single-tone sinusoids, as shown in (4). Thus, the time delay parameter estimation problem of the received signal $y(t)$ is converted to a frequency estimation problem of the transformed sinusoids $d(t)$.

\section{B. Received Signal with I/Q Imbalance}

Since millimetre-wave radar systems including direct conversion use extremely high frequencies, they are associated with the I/Q imbalance. In (3), we assume that the reference chirp signal $r(t)$ has a perfect I/Q balance. For an analysis of transformed signals perturbed by I/Q imbalance, we assume that the reference chirp signal $r^{\prime}(t)$ has the I/Q imbalance. The output of the reference $r^{\prime}(t)$ with the I/Q imbalance is expressed as

$$
r^{\prime}(t)=\cos (X)+j(1+\beta) \sin (X+\theta)
$$

where $X=\left(\omega_{c}+\omega_{s}\right) t+\left(t^{2}\right) / 2$ and $\theta$ and $\beta$ represent the error of the phase and the amplitude due to the I/Q imbalance, respectively. The conjugation of (5) can be changed as [10]

$$
r^{\prime}(t)^{*}=\xi^{+} e^{j X}+\xi^{-} e^{-j X}
$$

where

$$
\xi^{+}=1 / 2\left\{1-(1+\beta) e^{j \theta}\right\}
$$

and $\xi^{-}=1 / 2\left\{1+(1+\beta) e^{-j \theta}\right\}$. After de-chirping and low-pass filtering, it is determined that the transformed signals $d_{\mathrm{IQ}}(t)$ due to the I/Q imbalance have a sinusoidal waveform such that

$$
d_{\mathrm{IQ}}(t)=a \times\left\{\xi^{-} e^{\dot{m}(t)}+\xi^{+} e^{-\dot{m}(t)}\right\}+z(t) .
$$

The received signal perturbed by I/Q imbalance $d_{\mathrm{IQ}}(t)$ can be represented by a sum of the complex scaled desired signal and interfering signal in the imaginary frequency of the desired signal.

\section{Proposed PReprocessing TeChNiQUe}

The proposed method is composed of a two-step approach for the compensation of the I/Q imbalance error in order to estimate the single-tone frequency.

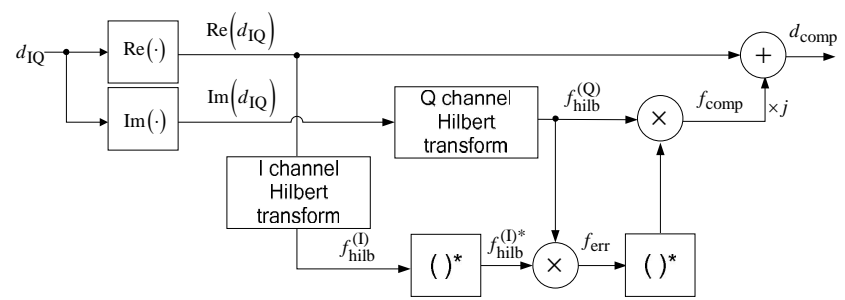

Fig. 1. I/Q imbalance compensator.

\section{A. Preprocessing Technique for I/Q Imbalance Compensation}

The proposed preprocessing technique for the I/Q imbalance compensation is shown in Fig. 1. The discrete time model of $d_{\mathrm{IQ}}(t)$ received through analog-to-digital converter (ADC) can be described such that

$$
\begin{gathered}
d_{\mathrm{IQ}}[n]=\xi^{-} a e^{\dot{m}\left(n T_{S}\right)}+\xi^{+} a e^{-\dot{m}\left(n T_{S}\right)}+ \\
+z\left(n T_{S}\right),
\end{gathered}
$$

where $n=0, \ldots, N-1, T_{\mathrm{s}}$ denotes the sample period and $N$ represents the number of samples per $T_{\text {sym }}$. In addition, in order to clarify the signal model with the I/Q imbalance, the following explanation omits the noise term, $z\left[n T_{\mathrm{s}}\right]$, in (8) for simplicity. $\operatorname{Re}\left(d_{\mathrm{IQ}}[n]\right)$ and $\operatorname{Im}\left(d_{\mathrm{IQ}}[n]\right)$ in (8) are fed into Hilbert transform functions of the $\mathrm{I}$ and $\mathrm{Q}$ channels, respectively. Here, $\operatorname{Re}()$ and $\operatorname{Im}($ ) means the real part of the complex signal and the imaginary part of the complex signal, respectively. The goal of the Hilbert transform is to generate a complex-valued $N$-point signal from the real-valued signal as shown in [11]. In the Hilbert transform of the I channel, $\operatorname{Re}\left(d_{\mathrm{IQ}}[n]\right)$ is transformed by using $N$-point DFT such that

$$
D_{\mathrm{IQ}}^{(\mathrm{I})}[k]=\sum_{n=0}^{N-1} \operatorname{Re}\left(d_{\mathrm{IQ}}[n]\right) e^{-j \frac{2 \pi k n}{N}},
$$

where $k=0,1, \ldots, N-1$. After DFT, an $N$-point one-sided spectrum is obtained via

$$
F_{\mathrm{hilb}}^{(\mathrm{I})}[k]=\left\{\begin{array}{l}
D_{\mathrm{IQ}}^{(\mathrm{I})}[k], \text { for } k=0, \\
2 D_{\mathrm{IQ}}^{(\mathrm{I})}[k], \text { for } 1 \leq k \leq \frac{N}{2}-1, \\
D_{\mathrm{IQ}}^{(\mathrm{I})}[k], \text { for } k=\frac{N}{2}, \\
0, \text { for } \frac{N}{2}+1 \leq k \leq N-1 .
\end{array}\right.
$$

To compute the inverse DFT (IDFT) of the $N$-point onesided spectrum $F_{\text {hilb }}^{(\mathrm{I})}[k]$ the complex analytic signal is computed by

$$
f_{\text {hilb }}^{(\mathrm{I})}[n]=1 / N \sum_{k=0}^{N-1} F_{\text {hilb }}^{(\mathrm{I})}[k] e^{j \frac{2 \pi k n}{N}},
$$


where for $n=0,1, \ldots, N-1$. The Hilbert transform of the Q channel from the imaginary-valued signal uses a procedure identical to that of the Hilbert transform of the I channel. And then, by multiplying $f_{\text {hilb }}^{(\mathrm{I}) *}[k]$ and $f_{\text {hilb }}^{(\mathrm{Q})}[k]$ obtained from the Hilbert transform of the I and Q channels, respectively, the $k$-th error term $f_{\text {err }}[k]=(1+\hat{\varepsilon}) e^{j \hat{\theta}}$ induced due to the $\mathrm{I} / \mathrm{Q}$ imbalance is achieved. After $f_{\text {err }}{ }^{*}[k]$ is multiplied by $f_{\text {hilb }}^{(\mathrm{Q})}[k]$, the $k$-th compensated signal $f_{\text {comp }}[k]$ is obtained. Finally, the $k$-th compensated complex signal $d_{\text {comp }}[k]$ is acquired from the sum of $\operatorname{Re}\left(d_{\mathrm{IQ}}[k]\right)$ and $j f_{\text {comp }}[k]$ i.e. $d_{\text {comp }}[k]=\operatorname{Re}\left(d_{\mathrm{IQ}}[k]\right)+j f_{\text {comp }}[k]$.

\section{B. TOA Estimation by ESPRIT}

Using the compensated signal $d_{\text {comp }}[k]$ with $N$ samples, the autocorrelation matrix $\boldsymbol{R}_{\mathrm{dd}}$ of $L$ by $L$ is defined as

$$
\mathbf{R}_{\mathrm{dd}}=\sum_{n=0}^{N-L} \mathbf{d}_{\mathrm{comp}} \mathbf{d}_{\mathrm{comp}}^{H}
$$

where the sequence $\boldsymbol{d}_{\text {comp }}=\left[d_{\text {comp }}[n], \ldots, d_{\text {comp }}[n+L-1]\right]^{\mathrm{T}}$ with length $L,()^{H}$ and ()$^{T}$ is the Hermitian transpose and transpose, respectively. The eigenvalue decomposition (EVD) of $\boldsymbol{R}_{\mathrm{dd}}$ has a form given by

$$
\mathbf{R}_{\mathrm{dd}}=\left[\begin{array}{ll}
\mathbf{S} & \mathbf{G}
\end{array}\right]\left[\begin{array}{cccc}
\lambda_{0} & 0 & \vdots & 0 \\
0 & \lambda_{1} & \ddots & \vdots \\
\vdots & \ddots & \ddots & 0 \\
0 & \cdots & 0 & \lambda_{L-1}
\end{array}\right]\left[\begin{array}{l}
\mathbf{S}^{*} \\
\mathbf{G}^{*}
\end{array}\right]
$$

where the signal eigenvector matrix $S=\left[\boldsymbol{s}_{0}, \ldots, \boldsymbol{s}_{M-1}\right]$ contains $M$ eigenvectors which span the signal subspace of the correlation matrix, the noise eigenvector matrix $\boldsymbol{G}=\left[\boldsymbol{g}_{0}, \ldots\right.$, $\left.\boldsymbol{g}_{L-M-1}\right]$ indicates $L-M$ eigenvectors spanning the noise subspace of the correlation matrix, and $\lambda_{n}$ denotes the $n$-th eigenvalues of $\boldsymbol{R}_{\mathrm{dd}}$. The largest $M$ eigenvalues of $\lambda_{0}, \ldots, \lambda_{M-1}$ correspond to the $M$ eigenvectors of $S$. The other eigenvalues $\lambda_{M}, \ldots, \lambda_{L-1}$ correspond to the eigenvectors of $\boldsymbol{G}$ such that $\lambda_{M}=\ldots=\lambda_{L-1}=\sigma^{2}$. Let us define the $\boldsymbol{S}_{1}$ and $\boldsymbol{S}_{2}$ matrices, which is $\boldsymbol{S}_{1}=\left[\begin{array}{ll}\boldsymbol{I}_{L-1} & \boldsymbol{0}_{1 \times L-1}\end{array}\right] \boldsymbol{S}, \boldsymbol{S}_{2}=\left[\begin{array}{lll}\boldsymbol{0}_{1 \times L-1} & \boldsymbol{I}_{L-1}\end{array}\right] \boldsymbol{S}, \boldsymbol{I}_{\mathrm{M}}$ denotes $M \times M$ identity matrix and $\boldsymbol{0}_{\mathrm{M} \times \mathrm{N}}$ means $M \times N$ zero matrix. The sub-matrices, as shown in earlier work [12], are factorized by:

$$
\left\{\begin{array}{l}
\mathbf{S}_{1}=\mathbf{A}_{1} \mathbf{C} \\
\mathbf{S}_{2}=\mathbf{A}_{1} \mathbf{D C}=\mathbf{S}_{1} \mathbf{C}^{-1} \mathbf{D C}=\mathbf{S}_{1} \phi
\end{array}\right.
$$

where $\boldsymbol{A}_{1}=\left[\begin{array}{ll}\boldsymbol{I}_{L-1} & \boldsymbol{0}_{1 \times L-1}\end{array}\right] \boldsymbol{A}, \boldsymbol{A}_{2}=\left[\begin{array}{ll}\boldsymbol{0}_{1 \times L-1} & \boldsymbol{I}_{L-1}\end{array}\right] \boldsymbol{A}, \boldsymbol{A}=\left[\begin{array}{l}\boldsymbol{a}\left(\omega_{0}\right) \ldots \\ \text { a }\end{array}\right.$ $\left.\boldsymbol{a}\left(\omega_{M-1}\right)\right], \boldsymbol{a}\left(\omega_{m}\right)=\left[1 e^{-j \omega_{m}} \ldots e^{-j(L-1) \omega_{m}}\right]^{\mathrm{T}}, \omega_{m}=\mu \tau_{m} T_{\mathrm{s}}, \boldsymbol{D}=\operatorname{diag}\left[\delta_{0}\right.$ $\left.\ldots \delta_{M-1}\right], \operatorname{diag}()$ means the diagonalization of the matrix, $\delta_{m}$ denotes the frequency of the transformed complex exponential signal for the $m$-th time delay of the received signal i.e. $\delta_{m}=e^{-j \omega_{m}}$ and $\mathbf{C}$ denotes the non-singular transformation matrix of $M$ by $M$. In order to obtain $\delta_{m}$, the eigenvalues of $\phi$ is used such as $\phi=\mathbf{C}^{-1} \mathbf{D C}$. Thus, $\phi$ is expressed such that

$$
\phi=\left(\mathbf{S}_{1}^{*} \mathbf{S}_{1}\right)^{-\mathbf{1}} \mathbf{S}_{1}^{*} \mathbf{S}_{2}
$$

Among several $\phi \mathrm{s}$, the first time delay of received signal $\hat{\tau}_{0}$ is determined by the first eigenvalue of $\phi$ such that

$$
\hat{\tau_{0}}=-\frac{1}{\mu T_{s}} \angle\left(\delta_{0}\right)
$$

where $\angle(\cdot)$ means the phase angles for a complex signal.

\section{PERformance ANAlysis OF RMSE AND CRLB}

In this section, the reduced root-mean-square error (RMSE) of the estimator for the received signal distorted by the I/Q imbalance, the ratio of the RMSE of the proposed method to the RMSE of the signal perturbed by the I/Q imbalance and the Cramer-Rao lower bound (CRLB) are derived. The distorted signal, $d_{\mathrm{IQ}}[n]$, leads to a signal to noise ratio (SNR) reduction of the received signal. From (7), the distorted signal $d_{\mathrm{IQ}}[n]$ is divided into the negative frequency and the positive frequency. Compared to (4), the amplitude of the negative frequency decreases with $\xi^{-}$. Therefore, the reduced SNR $\Delta$ due to $\xi^{-}$can be expressed as follows

$$
\Delta=20 \log _{10}\left(\xi^{-}\right)
$$

In the AWGN channel, the RMSE of the estimated TOA for the signal perturbed due to the I/Q imbalance $\delta_{\mathrm{IQ}}$ can be expressed as in (17) from earlier work [6] with the SNR which denotes as $\gamma$ i.e. $\gamma=a^{2} / \sigma^{2}$ such as

$$
\delta_{\mathrm{IQ}} \approx \frac{1}{\mu N T_{s}} \sqrt{\frac{1}{10^{0.1 \times(\gamma+\Delta)}}}
$$

The RMSE of the proposed method has similar performance comparing with the ideal ESPRIT because the proposed method removes the effect of the I/Q imbalance. The ratio of the RMSE of the proposed method to the RMSE of the signal perturbed due to an I/Q imbalance $R$ can be formulated such that

$$
R=\sqrt{\frac{1}{10^{0.1 \gamma}}} / \sqrt{\frac{1}{10^{0.1(\gamma+\Delta)}}}=\sqrt{10^{0.1 \Delta}}=\left|\xi^{-}\right| .
$$

Therefore, the RMSE of the proposed method is improved with $\left|\xi^{-}\right|$.

In the CRLB, the ESPRIT-based TOA estimation is based on a single-tone sinusoid signal. For the received signal in (4) in the AWGN channel, the CRLB for the ESPRIT-based TOA estimation $\delta_{\tau}$ is derived from an earlier result [10] such that

$$
\delta_{\tau}^{2} \geq \frac{6}{2 \pi \gamma N\left(N^{2}-1\right) \mu^{2} T_{s}^{2}}
$$

\section{Simulation Results}

We present Monte-Carlo simulation results averaged over 10,000 estimates to verify the performance of the proposed 
algorithm. The TOA performance of the proposed algorithm is compared with those by conventional algorithms, in this case the ESPRIT-based TOA estimation algorithm, in a variety of the I/Q imbalance cases. This letter only takes into account the RMSE for single-tone frequency. For the proposed and conventional algorithms, their RMSEs are defined such that $\sqrt{1 / N \sum_{n=1}^{N}\left\{\left(\tau_{1, n}-\tau_{1}\right)^{2}\right\}}$, where $\bar{\tau}_{1, n}$ is the first time delay estimation of the $n$-th Monte-Carlo trial and $N$ is 10,000. In the following simulations, we set the parameters as shown in Table I.

TABLE I. SIMULATION PARAMETERS FOR AWGN CHANNEL.

\begin{tabular}{|c|c|}
\hline Parameter & Value \\
\hline & $7.3158 \times 10^{12}$ \\
\hline$T_{\mathrm{s}}$ & $31.25 \mathrm{~ns}$ \\
\hline$T_{\mathrm{sym}}$ & $1.1875 \mathrm{~s}$ \\
\hline
\end{tabular}

In Fig. 2, the proposed algorithm is analysed for the RMSE of the estimated TOA for the signal perturbed due to the I/Q imbalance and compared to other algorithms and CRLB values. We compare the RMSE of the ideal ESPRIT algorithm with RMSE of the proposed estimator in TOA. Because the analysis assumes a high SNR, the analysed RMSE and the simulation results for the proposed algorithms are not well matched in SNR areas below $5 \mathrm{~dB}$ in Fig. 2.

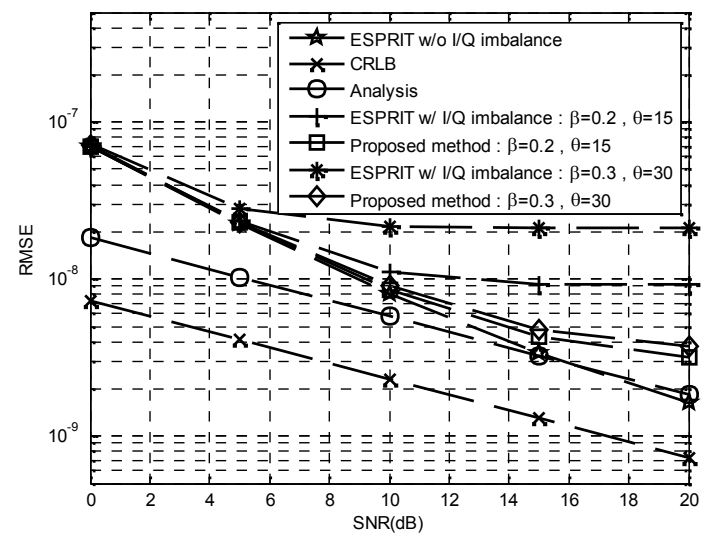

Fig. 2. Simulation and analytic results of TOA in the AWGN channel.

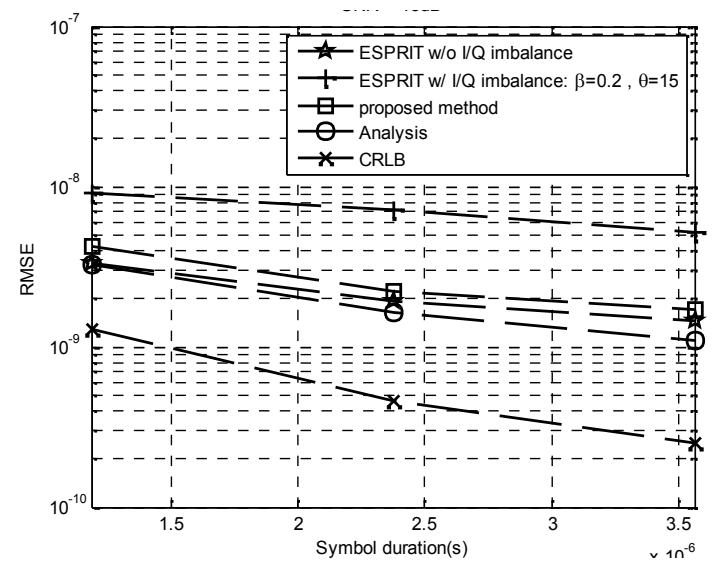

Fig. 3. Simulation and analytic results of TOA with various values of $T_{\text {sym }}$ with $\mathrm{SNR}=15 \mathrm{~dB}$.

For the received signals including I/Q imbalance error, the RMSE of the proposed algorithm is nearly identical to the ideal case, while the ESPRIT method with I/Q imbalance becomes saturated from the point when SNR $=10 \mathrm{~dB}$. Figure 3 shows the RMSE values of various estimators as the number of samples $N$ increases. Regarding the change of the sample number $N, N$ increases to 114 such that $T_{\text {sym }}=$ 3.56 s. When $N$ increases, the RMSE of the proposed estimator improves. In particular, for the proposed estimator, when $N$ changes from 38 to 114 , the RMSE characteristics improve by more than twofold, showing a change from $4.25 \mathrm{e}-9$ to $1.71 \mathrm{e}-9$.

\section{EXPERIMENTS}

In a real situation, to verify the robustness of the proposed method, a variety of experiments are performed in an anechoic chamber located at Daegu-Gyeongbuk Institute of Science \& Technology (DGIST) in Korea. In Fig. 4, the chamber is built for wireless signals of $8 \mathrm{GHz}$ to $110 \mathrm{GHz}$. The dimensions are $10 \mathrm{~m}(\mathrm{~L}) \times 5 \mathrm{~m}(\mathrm{~W}) \times 4 \mathrm{~m}(\mathrm{H})$. As shown in Fig. 4, moving targets can move back and forth for our experiments. We develop the radar verification equipment as shown in Fig. 5. By an external signal from the DSP board, the transmitted signal of the RF transceiver is generated. The received signals, which are called as beat signals, are captured by a Tektronix TDS8200 sampling oscilloscope. The captured data was used to verify the proposed algorithm as described in the next section.

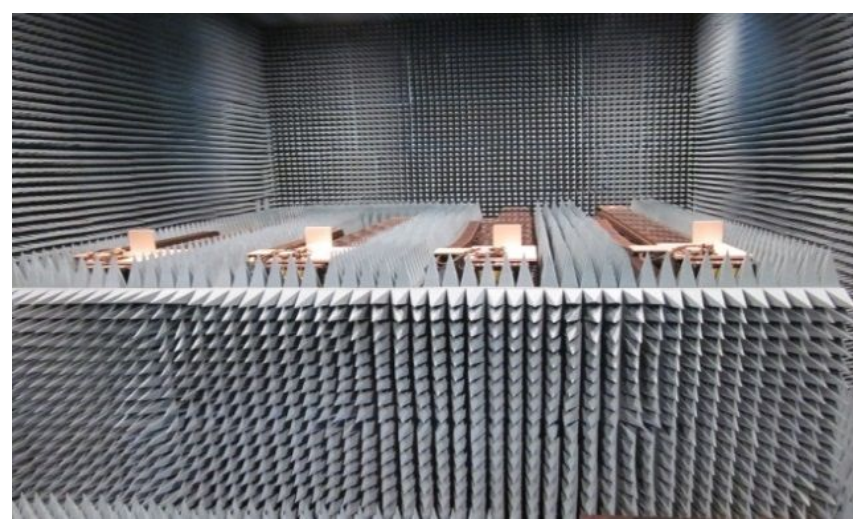

Fig. 4. Photo of the chamber room at DGIST.

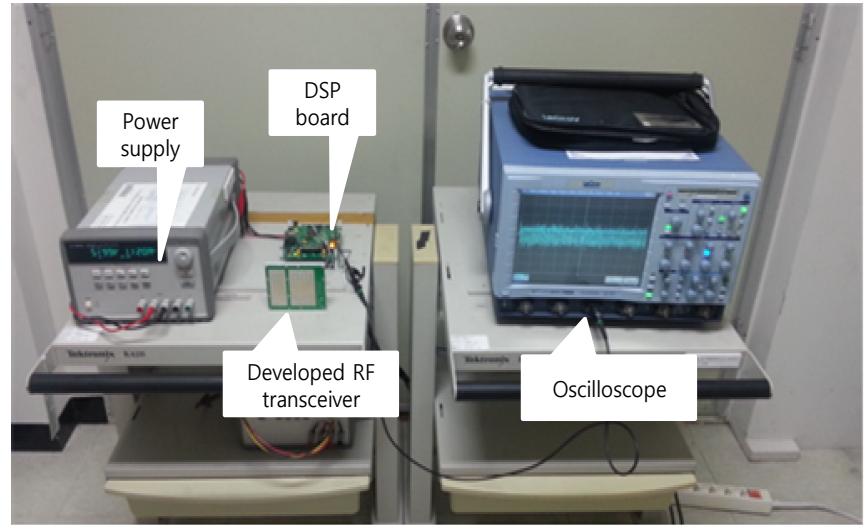

Fig. 5. Measurement setup of the $24 \mathrm{GHz}$ FMCW radar.

\section{A. The Description of RF Transceiver}

A block diagram of the $24 \mathrm{GHz}$ FMCW radar system is shown in Fig. 6. The system is consisted of a transmitter, a transmitting antenna of multiple elements, a receiving antenna, and a receiving channel. The transmitter generates 
FMCW signals which have a chirp signal. The initial transmission time of each sweep is controlled by the DSP board. A photograph of the $24 \mathrm{GHz}$ radar system is shown in Fig. 7.

The transmitter comprises a voltage-controlled oscillator (VCO), frequency synthesizer and a $26 \mathrm{MHz}$ oscillator. To generate the FMCW source, a frequency synthesizer controls the input voltage of the VCO. An RF signal is spread to the
TX antenna and receiver mixer via the power divider.

The receiver contains a LNA, a mixer, a high-pass filter (HPF) and a low-pass filter (LPF). The receiver has an overall noise figure of $8 \mathrm{~dB}$. The gain and noise figure of the LNA are $14 \mathrm{~dB}$ and $2.5 \mathrm{~dB}$, respectively. In order to generate the beat signal, an RF signal is down-converted by the mixer. The measured $3 \mathrm{~dB}$ cutoff frequency of the HPF and the LPF are about $13 \mathrm{KHz}$ and $2 \mathrm{MHz}$, respectively.

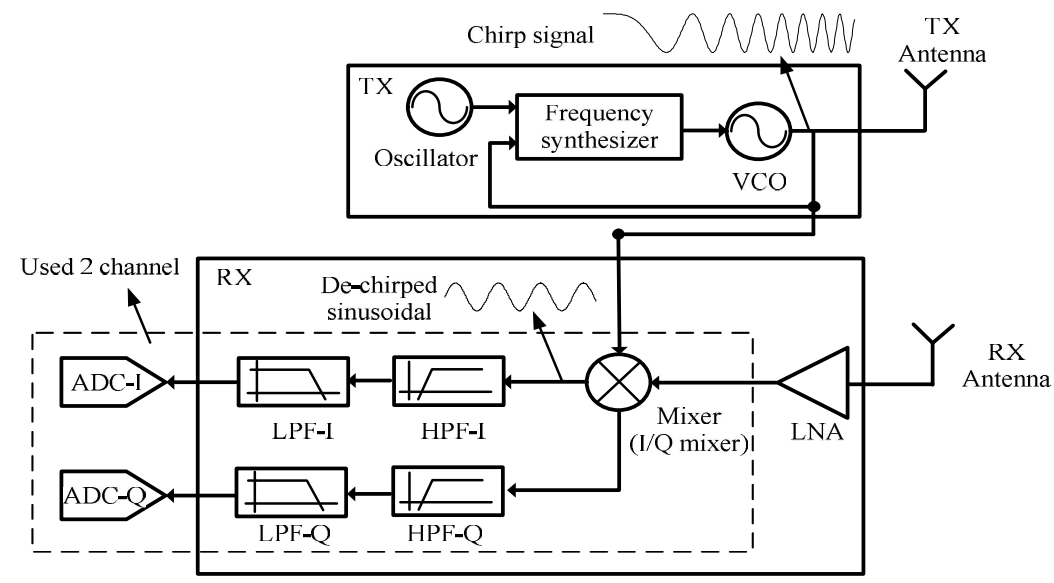

Fig. 6. Block diagram of the $24 \mathrm{GHz}$ FMCW radar system.

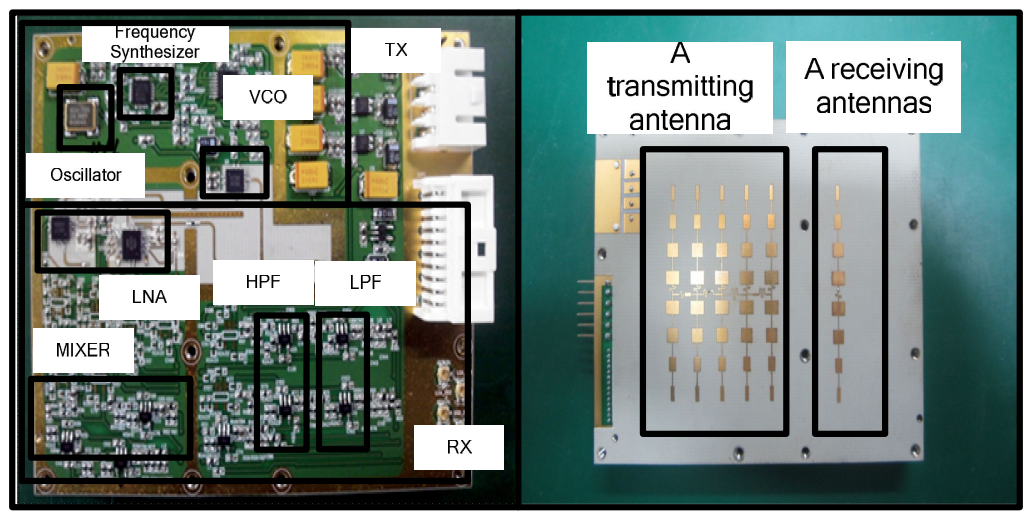

Fig. 7. Photograph of the $24 \mathrm{GHz}$ FMCW radar system.

\section{B. Antennas}

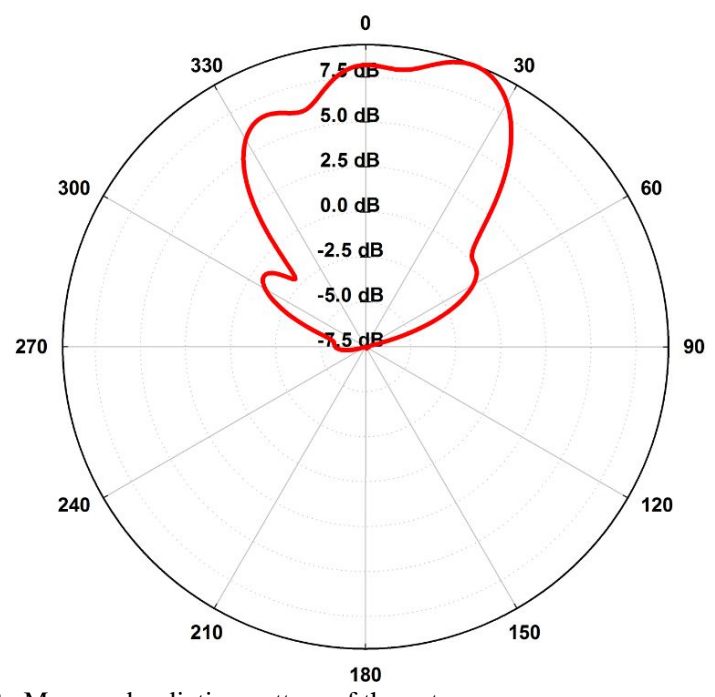

Fig. 8. Measured radiation pattern of the antenna.

An antenna architecture in the receiving antenna is a microstrip-patch antenna. This microstrip-patch antenna has a gain of $15.6 \mathrm{dBi}$ and a $3-\mathrm{dB}$ beamwidth of $24^{\circ}$. The microstrip-patch antenna of the same type is mounted on the components of the transmitting antenna. The measured radiation pattern of the antenna with horizontal polarization is shown in Fig. 8.

\section{Experiments}

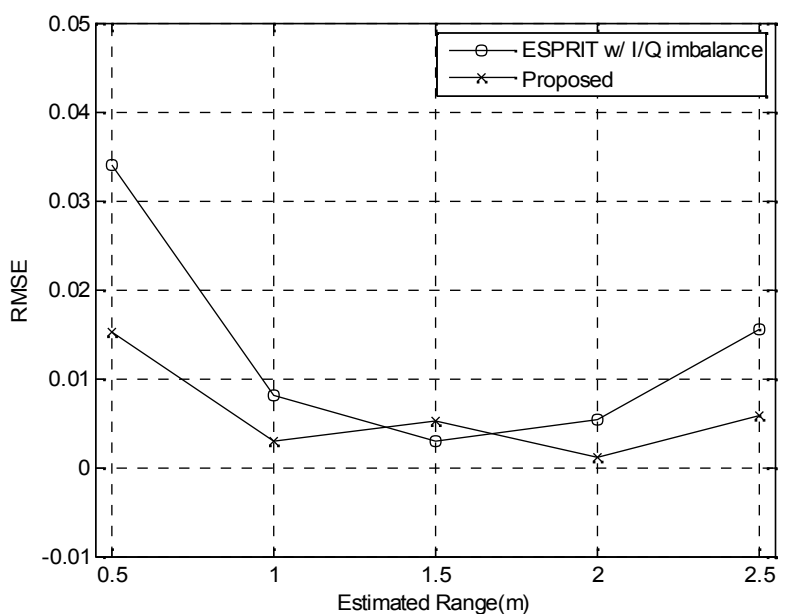

Fig. 9. Range map of the proposed method for the target located at ranges of $0.5 \mathrm{~m}$ and $2.5 \mathrm{~m}$. 
In chamber environment, we carried out single-target experiments. In order to evaluate the performance of the proposed algorithm, the conventional ESPRIT with I/Q imbalance are compared. In this single-target scenario, three different single-target scenarios are performed. When a target was located at ranges of $0.5 \mathrm{~m}$ and $2.5 \mathrm{~m}$ relative to the FMCW RF module, the range RMSE of the proposed method was obtained as shown in Fig. 9. In Fig. 9, the range results of the proposed estimator are found at $0.47 \mathrm{~m}$, $0.72 \mathrm{~m}, 1.4 \mathrm{~m}, 1.75 \mathrm{~m}$ and $2.19 \mathrm{~m}$, which the mean of ranging RMSE is $0.0061 \mathrm{~m}$. Compared to the range map of the ESPRIT with I/Q imbalance in Fig. 9, it can be seen that the RMSE of the proposed estimator, which the mean of ranging RMSE is $0.0132 \mathrm{~m}$, is better than that of the conventional estimator.

\section{CONCLUSIONS}

For robust ESPRIT-based single-tone frequency estimations considering I/Q mismatch, we proposed an effective preprocessing technique. The estimation performance of the proposed preprocessing technique in a single target is analysed for the RMSE of the estimated TOA for the signal perturbed due to the I/Q imbalance and compared with Monte-Carlo simulation results. The proposed method is achieved similar performance of the ideal ESPRIT method without the I/Q imbalance with various parameters.

\section{REFERENCES}

[1] K. W. K. Lui, H. C. So, "Modified Pisarenko harmonic decomposition for single-tone frequency estimation", IEEE Trans. on Signal Process., vol. 56, no. 7, pp. 3351-3356, 2008. [Online]. Available: http://dx.doi.org/10.1109/TSP.2008.920486

[2] H. C. So, F. K. W. Chan, W. Sun, "Subspace approach for fast and accurate single-tone frequency estimation", IEEE Trans. on Signal Process., vol. 59, no. 2, pp. 827-831, 2011. [Online]. Available: http://dx.doi.org/10.1109/TSP.2010.2090875

[3] E. Jose, M. Adams, J. S. Mullane, N. M. Patrikalakis, "Predicting millimeter wave radar spectra for autonomous navigation", IEEE Sensors Journal, vol. 10, no. 5, pp. 960-971, 2010. [Online]. Available: http://dx.doi.org/10.1109/JSEN.2009.2037013

[4] R. Svitek, S. Raman, "DC offsets in direct-conversion receivers: characterization and implications", IEEE Microwave Mag., vol. 6, no. 3, pp. 76-86, 2005. [Online]. Available: http://dx.doi.org/ 10.1109/MMW.2005.1511916

[5] X. Li, K. Pahlavan, "Super-resolution TOA estimation with diversity for indoor geolocation", IEEE Trans. on Wireless Commun., vol. 3 , pp. 224-234, 2004. [Online]. Available: http://dx.doi.org/ 10.1109/TWC.2003.819035

[6] T. K. Sarkar, O. Pereira, "Using the matrix pencil method to estimate the parameters of a sum of complex exponentials", IEEE Antennas Propagat. Mag., vol. 37, pp. 48-55, 1995. [Online]. Available: http://dx.doi.org/10.1109/74.370583

[7] S. Marcos, A. Marsal, M. Benidir, "The propagator method for source bearing estimation", Signal Process., vol. 42, no. 2, pp. 121-138, 1995. [Online]. Available: http://dx.doi.org/10.1016/01651684(94)00122-G

[8] A. N. Lemma, A. J. Vanderveen, E. F. Deprettere, "Multiresolution ESPRIT Algorithm", IEEE Trans. on Signal Process., vol. 47, pp. 1722-1726, 1999. [Online]. Available: http://dx.doi.org/10.1109/ 78.765149

[9] D. Oh, M. Kwak, J. Chong, "A subspace-based two-way ranging system using a Chirp Spread Spectrum modem, robust to frequency offset", IEEE Trans. on Wireless Commun., vol. 11, no. 4, pp. 14781487, 2012. [Online]. Available: http://dx.doi.org/10.1109/TWC. 2012.030512.111044

[10] I. Sohn, E. Jeong, Y. H. Lee, "Data-aided approach to I/Q mismatch and DC offset compensation in communications receivers", IEEE Commun. Lett., vol. 6, no. 12, pp. 547-549, 2002. [Online]. Available: http://dx.doi.org/10.1109/LCOMM.2002.806451

[11] S. L. Marple, Jr., "Computing the discrete-time "analytic" signal via FFT", IEEE Trans. on Signal Process., vol. 47, no. 9, pp. 2600 2603, 1999. [Online]. Available: http://dx.doi.org/10.1109/78.782222

[12] D. Oh, J. Lee, J. W. Chong, "An effective pre-filtering method with a propagator for TOA-based range estimation using chirp signal", IEEE Commun. Lett., vol. 15, no. 9, pp. 929-931, 2011. [Online]. Available: http://dx.doi.org/10.1109/LCOMM.2011.072911.102217 